NASA Technical Memorandum 100148

\title{
Ray Propagation Path Analysis of Acousto-Ultrasonic Signals in Composites
}

\author{
(NASA-TH-100148) RAY PROPAGATION PATH \\ ANA LYSIS OF ACOUSTO-ULTRASONIC SIGNALS IN \\ COUPOSITES (NASA) $20 \mathrm{p}$ Avail: NTIS HC \\ $\mathrm{AC} 2 / \mathrm{MF}$ AO 1 \\ CSCl 140 \\ N87-25589 \\ Unclas \\ G3/38 0085597
}

Harold E. Kautz

Lewis Research Center

Cleveland, Ohio

Prepared for

Acousto-Ultrasonics: Theory and Application sponsored by NASA Lewis Research Center Blacksburg, Virginia, July 12-15, 1987 
RAY PROPAGAIION PATH ANAYLSIS OF ACOUSTO-ULTRASONIC SIGNALS IN COMPOSITES

\author{
Harold E. Kautz \\ National Aeronautics and Space Administration \\ Lewis Research Center \\ Cleveland, Ohio 44135
}

\title{
SUMMARY
}

The most important result of this work was the demonstration that acoustoultrasonic (AU) energy introduced into a laminated graphite/res in propagates by two modes through the structure. The first mode, along the graphite fibers, is the faster. The second mode, through the resin matrix, besides being slower is also more strongly attenuated at the higher frequencies. This demonstration was accomplished by analyzing the time and frequency domain of the composite $A U$ signal and comparing them to the same for a neat resin specimen of the same chemistry and geometry as the composite matrix.

The separation of the frequency spectra of propagation modes was accomplished on a broad scale. Analys is of the fine structure of AU spectra was accomplished by various geometrical strategies. It was shown that the multitude of narrow peaks associated with AU spectra are the effect of the many pulse arrivals in the signal. Differences between pulse arrivals leads to characteristic times and interference peaks in the spectrum. The shape and distribution of the peaks is mainly determined by the condition of nonnormal reflections of ray paths. Nonnormal reflections causes the characteristic times to not be simply related and changes discrete times to distributions of times. A cepstrum analysis was employed which can be useful in detecting characteristic times.

Analysis of propagation modes can be accomplished while ignoring the fine structure. If the fine structure of an AU signal is to be studied, however, it is necessary to be aware of possible contributions from unwanted sources such as multiple reflections in dry couplant pads.

\section{INTRODUCTION}

It has been established that the stress-wave factor (SWF) calculated from acousto-ultrasonic (AU) signals is sensitive to mechanical properties in composite structures (refs. 1 to 13). In particular, it was shown (ref. 1) that SWF was sensitive to interlaminar shear strength (ISS) in graphite/epoxy.

More recent studies have shown that analysis of $A U$ wave propagation paths in relatively thick specimens taken from filament wound composite (FWC) structures is useful for predicting ISS (refs. 14 and 15). This analys is led to the prediction that the relation between SWF and ISS would be strongest in certain partitions of the AU signal in the time domain. The propagation path analysis correctly predicted, for the material and geometry under consideration, that signals arriving about 15 to $20 \mu \mathrm{s}$ into the waveform would carry the least ambiguous information on laminar interfaces.

The analysis in references 14 and 15 also included frequency domain partitioning. The ray path model employed correctly identified time segments of 
interest, but made no predictions about frequency. It was evident from this work, however, that there was dependence on frequency. Indeed, partitioning of the frequency domain seemed at least as useful as that of the time domain in revealing good correlation between SWF and ISS. It seems appropriate, therefore, to examine factors that may shape the frequency spectrum of composite $A U$ waveforms.

The most striking finding in the present work is the identification of particular propagation paths in a graphite/epoxy panel by comparison of the AU signal with that collected in an otherwise similar panel composed of the epoxy resin alone. Beyond this, the rest of this paper attempts to identify the source of other features that are commonly encountered with AU signals.

\section{ANALYSIS}

\section{Attenuation Effects}

It is evident that many factors are involved in the shaping of an AU frequency spectrum. The two most obvious are the response of the transducers and the attenuating characteristics of the composite. Materials tend to attenuate high frequency ultrasonic waves more than low frequency waves (ref. 16, p. 89). The attenuating effect is especially strong in fiber/polymer composites. For this reason one expects that the output spectrum obtained at the receiving transducer will center at a lower frequency than the input spectrum introduced by the sending transducer.

\section{Multiple Path Effects}

It has been pointed out (ref. 16) that layered interfaces along an ultrasonic ray path give rise to multiple arrival times for propagating waves. These produce interference effects that appear as peaks in the frequency spectrum. Another source of the same kind of interference effects is the arrival of signals from along different propagation paths (ref. 17).

In each of these interference effects all the signal arrivals at the receiver are from a common input pulse. Extremes (peaks or troughs), in the spectrum will be centered at frequencies, $f$, where $f$ is related to the characteristic time, $T$, between the arrivals as:

$$
T=\frac{n}{f}
$$

and also as:

$$
T=\frac{\left(n+\frac{1}{2}\right)}{f}
$$

Where $n$ is an integer (see appendix $A$ ). If equation (1) produces a maximum then equation (2) will be minimum. The maximum-minimum conditions can be reversed depending on the phase relation between the pulses.

The interference effects are oscillations in the frequency domain. Just as the frequency spectrum is obtained by taking the magnitude of the fourier 
transform of the original time domain signal, the fourier transform of the spectrum can be used to obtain a function sometimes called the cepstrum. This cepstrum will exhibit resonance at the characteristic times $T$. This may be useful in identifying characteristic times.

\section{EXPERIMENTAL}

\section{Materials}

AU signals were collected on a $0.25 \mathrm{~cm}$ thick, 30 by $30 \mathrm{~cm}, 26 \mathrm{ply}$, graphite/epoxy resin panel. The resin matrix was 1500 molecular weight poly merization of monomeric reactants (PMR-15) with the ester of pyromellitic dianhydride (PMDE ester). The graphite lay-up was alternate $0 / 90$ degree orientations of Celion 6000 unfinished, $7 \mu \mathrm{m}$ diameter, fiber. AU signals were also collected on a $0.25 \mathrm{~cm}$ thick, 30 by $30 \mathrm{~cm}$, PMR pure resin panel of the same chemistry as described composite matrix.

Signals were also collected on a $0.88 \mathrm{~cm}$ thick, approximately 12 by $8 \mathrm{~cm}$, specimen of lucite. The lucite was used to simulate some of the ultrasonic response characteristic of graphite/epoxy without the presence of internal reflections at lamina boundries.

\section{Acousto-Ultrasonic System and Procedures}

In these experiments $A U$ signals were collected, digitized, and stored on disc. A block diagram of the data processing system is shown in figure 1 . Two $2.25 \mathrm{MHz}$ center frequency broad band transducers were used with $0.17 \mathrm{~cm}$ thick silcone rubber dry couplant pads and approximately $9 \mathrm{~N} / \mathrm{cm}^{2}$ pressure. The transducers were the immersion type with wearplate faces. The conditions are the same as for previous work reported in reference 15. An extensive spectral analysis was performed with the aid of the fast Fourier transform (FFT) algorithm. This analys is included calculation of frequency spectra for segments of the time domain signal. The FFI was performed on the Hanning windowed segment (ref. 18). The purpose of the Hanning window is to suppress artificial frequency components that are observed when a simple rectangular window is used to gate the segment of interest.

The data reported here from the composite and the resin panel were acquired by placing the $A U$ transducers at a point at about the center of one side of the 30 by $30 \mathrm{~cm}$ panels. This was found to be typical of data acquired at other positions on the panels.

Besides the AU data on the composite panels, waveform data was also collected with various other transducer configurations. These geometries were used to compare data from through-transmission signals at normal incidence to through transmission with off-set transducers and to the AU configuration. With the lucite specimen, immersion type $1 \mathrm{MHz}$ broad band sender and receiver were used. The $1 \mathrm{MHz}$ transducers on lucite produced a frequency range that simulated that of a pair of $2.25 \mathrm{MHz}$ transducers with the attenuating effect of a typical graphite/epoxy resin composite. Gel liquid couplant was used with the $1 \mathrm{MHz}$ transducer, lucite specimen experiments. 


\section{RESULTS}

\section{AU Comparison of Graphite/Epoxy Composite with Pure Epoxy Resin}

Figure 2 presents typical AU data for the graphite/epoxy panel and also for the panel of the epoxy alone. Figures $2(a)$ and (b) are time domain signals. Figure 2(a) indicates four partitions of interest in the composite signal marked $A, B, C$, and $D$. The signal for the resin in figure $2(b)$ is marked with the same partitions. Figures $2(c)$ and (d) are the frequency spectra of figures 2(a) and (b), respectively. Figures $2(e)$ through (1) are the Hanning windowed partitions of the four time domain signals from both specimens. Partition A is 2 to $17 \mu \mathrm{s}, B$ is 17 to $24 \mu \mathrm{s}, C$ is 24 to $48 \mu \mathrm{s}$, and $D$ is 48 to 100 Hs.

\section{Studies with Lucite and $1 \mathrm{MHz}$ Iransducers}

Figure 3 illustrates the geometry employed for one series of through transmission experiments performed on a lucite panel with two $1 \mathrm{MHz}$ trans ducers. In this case the transducers are lined up face to face so that the ultrasonic ray paths that pass through the lucite arrive at the receiving transducer at normal incidence.

Figure 4 is a series of through transmission signals collected in this manner. The column of figures on the left contains the time domain signals, the second column contains the frequency spectrum of signals, and the last column shows the cepstrum. In figure 1(a) the digitizer time base delay has been set to capture only the first pulse from the sender. Directly below in figure 4 (c) the delay is changed to capture the first pulse plus the first echo. Below that in figure $4(f)$ two echos are included so that a total of three signals are captured. Finally at the bottom of the first column in figure $4(i)$ all the detectable pulses are brought on the screen and captured.

In the next set of experiments the geometry of figure 3 is altered as indicated in figure 5 . The receiving transducer has been moved from the face to face configuration such that a direct ultrasonic ray path connecting the centers of the wear plates will make approximately a $\%^{\circ}$ angle with the normal to the contact plane.

Figure 6 shows waveforms collected with this arrangement. In figures 6(a) and (b) the digitizer time base has been set to capture only the first pulse from the sender. In figures $6(c)$ to $(e)$ all the detectable pulses are brought on screen and captured. The format of the presentation is the same as in figure 4; the first column shows the time domain signal, the second column shows the frequency spectrum, and the last column shows the cepstrum.

The transducer arrangement in figure 7 was used for the acousto-ultrasonic results in figure 8 . As with previous figures, 8(a) shows the time domain, 8 (b) shows the spectrum, and $8(c)$ shows the cepstrum.

Figure 9 data were collected with lucite and the normal incidence arrangement similar to that shown in figure 3. This time, however, one of the liquid couplant contacts is replaced by a $0.17 \mathrm{~cm}$ thick silicone rubber pad. This pad was typical of the kind used on both transducers in earlier AU work with graphile/epoxy resin composites. 


\section{DISCUSSION}

\section{Analysis of AU Spectra on Graphite/Epoxy and Pure Epoxy Resin}

The time domain representation of an $A U$ signal is the superposition of many pulse arrivals at the receiving transducer. The wide range of travel times among these pulses leads to the long time span associated with the signal. It seems likely that different time segments may have different spectra. $V$ isual inspection of the AU signal often suggests a dominance by relatively high frequency components near the beginning with a progression to lower frequency dominance towards the end. This might be explained by considering the effect of attenuation. Higher frequency waves are attenuated more than lower frequency waves. Any wave that travels a long path length with a given rate of attenuation will be more affected by this than an otherwise similar wave traveling a shorter path.

Some AU signals, however, suggest sharper changes in frequency dominance than seems likely from attenuation alone. This is the case with the waveform presented in figure 2(a). It exhibits what could be as many as four independent frequency distributions. Figure $2(c)$ indicates low frequency peaks at 0.3 and $0.5 \mathrm{MHz}$, and two high frequency peaks at about 1.0 and $1.5 \mathrm{MHz}$. The left hand member of each of the pairs of figures $2(e)$ to $(k)$ show the spectra of Hanning windowed partition of the time domain of the composite. Figures $2(e)$ and $(\mathrm{g})$ indicate that the 2 to $17 \mu \mathrm{s}$ and the 17 to $24 \mu \mathrm{s}$ partitions have a lmost the same spectrum. They are very strong in the 1.0 and the $1.5 \mathrm{MHz}$ peaks. Figure $2(\mathrm{k})$ shows that the 48 to $100 \mu \mathrm{s}$ partition is strong in the low frequency peaks. The intermediate partition of 24 to $48 \mu \mathrm{s}$ is shown in figure $2(i)$ to be a transition region. This result suggests at least two modes, or two mode types of propagation in this material. One mode passes through the specimen in a short time and retains frequencies up to the $1.5 \mathrm{MHz}$ region. The other takes relatively long and loses frequencies above about $0.5 \mathrm{MHz}$. The composite total AU signal, (figs. $2(a)$ and (c)), does not by itself give clear information concerning the nature of these two types of propagation.

The right hand member of each pair of graphs in figure 2 is an identical analysis as the left member except that it is with the $0.25 \mathrm{~cm}$ thick PMR epoxy resin panel. It is a specimen with no graphite fiber structure. Whal is most clear in the set of graphs is the absence of the high frequency, early arrival waves. This is evident by comparing figure $2(b)$ to $2(a)$ and then comparing figure $2(d)$ to $2(c)$. One is led to infer that the high frequency, early arrival mode in the composite is due to propagation on the graphite fibers.

For comparison with the composite, the resin AU signal has been analyzed in terms of the same, Hanning windowed, time partitions. Note that the bulk of the signal comes in the 24 to $48 \mu \mathrm{m}$ partition of figure $2(j)$. As with the composite in figure $2(i)$ it appears to exhibit a bimodal low frequency distribution. Unlike figure $2(i)$ there is little high frequency component. The similarity between the spectra of the resin and the low frequency components in the composite infers that the low frequency response in the graphite-epoxy is due to propagation through its epoxy resin component where the attenuation is high and the ultrasonic velocity is low relative to the fiber component. 


\section{Propagation Model for Composites}

Figure 10 illustrates a plausible propagation model for the figure 2 results. Figure 10(a) represents propagation through the pure resin. This is the simple case with the detected signal being shaped by the specimen only through attenuation of PMR and multiple reflection at the surfaces of the pane 1 .

Figure 10(b) represents the laminated composite panel. The resin path is still present but now there is an additional mode due to the fiber layers. This mode may travel on the fibers, possibly as transverse waves. Another possibility is that the fiber layers provide additional reflecting planes making the layers act as wave guides for the high frequency mode. Either way, the ultrasonic energy must get from the sending transducer to the fiber layers by first passing through the resin. It was noted during these experiments that touching the panel surface between the transducers with an energy absorbing medium strongly diminished the resin mode of propagation, but had little effect on the high frequency component. Thus it seems that once the ultrasonic energy gets into the fiber layers, the bulk of the high frequency energy travels along the fibers with some re-radiation occurring along the way.

Comparison of figures $2(a)$ and (b) shows that the resin mode, partition $D$, persists longer in the composite than in the resin alone. This mode is still present out to $100 \mu \mathrm{m}$ for the composite, but is essentially gone by $70 \mu \mathrm{m}$ for the neat resin. This seems to imply that the high frequency mode, while propagating internally, tends to radiate energy to the resin along the way thus enhancing the latter intensity. Note in figure 2(e) that the spec trum for the 2 to $17 \mu \mathrm{m}$ partition has a low frequency part that is in the region of the 48 to $100 \mu \mathrm{m}$ spectrum of figure $2(k)$. The fibers do carry low! frequency waves and re-radiate them to the resin. Figure 10 attempts to illus trate these ideas.

Not all graphite/epoxy structures may exhibit as sharp a time domain distinction of propagation modes as this one. It seems likely, however, that in principle the same propagation modes exist in all graphite/epoxy structures. Specimen geometry, especially thickness, can effect their relative importance. For example it has been shown that very thin specimens will be dominated by surface waves (ref. 7). Awareness of the present results may be useful in analyzing other geometries.

\section{Other Effects on the AU Spectra of Graphite/Epoxy Resin}

The analysis of AU spectra in figure 2 was carried out with attention to the overall structure in terms of major peaks in the frequency spectrum. What was ignored was a fine structure of spike like peaks which is especially obvious in the spectra of figures $2(c)$ and (d). The experiments discussed next are an investigation of factors that might produce the fine structure.

If we examine the AU spectra of the composite and the PMR epoxy resin in figure 2 the attenuation effects of the specimens is quite evident. The broad transducer resonance at $2.25 \mathrm{MHz}$ is not at all visible in the AU spectra. The specimen NU spectra are large in various frequency regimes. The regimes depend upon which material is examined, but in each case they are we 11 below $2.25 \mathrm{MHz}$. 
The overall shift to lower frequencies is expected on the bas is of attenuation. What is not explained is the presence of many spikes in the spectra. Neither the transducer response nor classical attenuation theory can explain this. However, it can be understood through examination of the effects of transducer positioning and of dry couplants on AU waveforms.

\section{Effects of Transducer Positioning}

Figures $4,6,8$, and 9 show waveforms collected with two immersion type, $1 \mathrm{MHZ}$, broad band transducers.

for figure 4 the transducers were in a face to face through transmission configuration. In figure $4(a)$ the time delay has been set to digitize only the first pulse that comes through. The spectrum in figure 4 (b) approximates a slightly skewed Gaussian distribution peaking near $1 \mathrm{MHz}$. The column of figures $f(c),(f)$, and $(i)$ show cases where progressively more echos are added to the captured signal. In figure $4(c)$ the time delay has been adjusted to digitize the initial arrival plus the first echo. Figure $4(f)$ contains the initial arrival plus two echos and figure $4(i)$ contains all the detectable arrivals. The center column of figure 4 shows the effect of multiple arrivals on the spectrum. The spectra display interference peaks that are reminiscent of the spikes in the AU spectra discussed earlier.

The interpretation of the peaks is simplest with the case of just two arrivals as with figure $4(d)$. The height of the peaks and depth of the troughs depends upon how much the first echo is attenuated relative to the in $i$ tial arrival. The locus of points for the minima is a curve of the difference in magnitudes of the two pulses as a function of frequency while the maxima fall on the curve of the sum of the amplitudes. This is illustrated by equations $(A 7)$ and $(A 8)$ of appendix $A$.

The frequency interval between peaks is the reciprocal of the round trip time between the signal arrivals in the first column. These characteristic times appear as the major peaks in the cepstrums of the last column. Note that since figure $A(c)$ has one characteristic time between arrivals, figure $4(e)$ has one peak. Figure $4(f)$ with two possible characleristic times between arrivals leads to figure $4(h)$ with two peaks. Figure $4(i)$ with all the detectable arrivals leads to figure $4(k)$ with a train of diminishing peaks which presumably obey equations (1) or (2).

The above analysis of pulse and echos produces only the characteristic times that are integer multiples of the round trip duration through the thick ness of the specimen. Characteristic times in AU signals will not be so simply related. In the simplest cases the times involved are for multiple reflections at nonnormal incidence. The times must be solved from more complex geometry of ray paths (refs. 14, 15, and 19). Besides this there is the effect of mode conversion at nonnormal reflection (ref. 16, pp. 476-481). Mode conversion mean's that not all rays travel with the same velocity.

Figures 5 and 6 illustrate a condition intermediate between simple normal incidence through transmission and the complex situation found in acoustoultrasonics. Here the transducers are still on opposite faces but they are off-set such that the ray path from the center of one wear plate to the center of the other makes an angle of approximately $77^{\circ}$ from normal. In figures $6(a)$ 
and (b), as in figures $4(a)$ and (b), only the first pulse, as best as it can be isolated, is digitized. The frequency spectrum in figure $6(b)$ is a relatively smooth curve with interference peaks separated by large frequency increments. These interference peaks did not appear in figure $4(b)$. In the present case we are probably seeing the effect of a distribution of characteristic times. The active area of the transducer wearplates is finite. For this reason there is a range of first pulse transit times for propagation between them.

For figures $6(c)$ to $(e)$ the total detectable signal was digitized. The signal looks much like an AU waveform, but still there are individual pulse arrivals that can be distinguished in figure $6(c)$. The frequency spectrum and cepstrum reveal that there are now many important frequency peaks and characteristic times for this signal. (It is interesting to do the experiment and watch the multiple reflections from the face to face geometry spread out in the time domain and change to different ray paths as the angle of these ray paths increases with the off-set sliding of the receiving transducer. In addition to this change there is the appearance, and gradual increase in importance, of new propagation paths associated with mode conversion at reflection.)

Figure 8 shows data obtained with the same transducers and specimen in an AU configuration, that is, with both transducers on the same side of the lucite. Now the arrival of individual pulses is much more obscure. The spectrum and cepstrum are more complex in structure. This signal, which has the spike like interference spectrum of a graphite/epoxy specimen, has been produced solely by positioning the transducers relative to each other similar to that shown in figure 1 .

The experiments described with figures 4,6 , and 8 have illustrated a step by step construction of the spectrum fine structure of an AU signal. The center column of figure 4 shows that the presence of multiple pulse arrival times leads to multiple peaks in the spectrum. But the peaks in figure 4 are broader than is typical for AU spectra. However when the propagation path of the rays is made nonnormal as in figure 6 the spectral peaks are found to become narrower. This is much more like the AU spectrum of figure $8(b)$ as we 11 as the composite and resin spectra in figures $2(c)$ and (d). This seems to show that this narrow, spike like, fine structure of the AU spectrum can be at least partly attributed to off-normal angles of reflection. off-normal reflection causes generation of mode conversion and characteristic times that have no simple arithmetic relation to each other. In addition to this, dis-crete characteristic times become distributions of times.

\section{Effect of Dry Couplants on AU Data}

The above experiments show that the frequency spectrum obtained from $A U$ signals can be simulated by the geometry of the specimen and transducer pos $i$. tions. It does not, however, prove that this is the only source of the peaks in the frequency spectrum. Figure 9 exhibits the results of an experiment designed to show the effect of silicone rubber pads. This thickness of this material was used as a dry couplant in several studies of graphite/epoxy composite structures (refs. 14 and 15). Comparing figure 9 (b) to figure $4(\mathrm{~b})$ one can conclude that there is an interference patlern introduced by the silicone rubber layer. It is likely that this is the result of multiple reflection within the pad. The cepstrum figure, $9(c)$, shows there are many characteris tic times present, with the most prominent being $6.7 \mu \mathrm{s}$. 


\section{CONCLUSIONS}

Acousto-ultrasonic energy introduced into a laminated graphite resin has been shown to propagate through the structure in two modes. The first mode is along the graphite fibers. This mode is the more rapid of the two. The secand mode is through the resin matrix. Besides being slower, the matrix mode is also the most strongly attenuated in the higher frequency regime.

Awareness of these differences in propagation speed and frequency distri.bution can be of use in separating the modes for study. It is likely that the analysis is applicable to other kinds of composites as well.

Studies of $A U$ in conjunction with material and mechanical properties should take advantage of this type of wave propagation mode analysis. Some modes will be more sensitive to particular properties than will others. Focus ing on the most appropriate mode can lead to higher reliability in using acousto-ultrasonics as a predictor for the property of interest.

The fine structure of narrow peaks in the frequency spectrum, typical of AU spectra, has been shown to be a manifestation of the characteristic times associated with the arrival of individual pulses at the receiving transducer. The times are not in general related as simple arithmetic ratios. They are not discrete but rather distributions of times. These features would seem to discourage attempts to analys is them. This fine structure is not likely to carry useful information on the material or mechanical properties of the specimen being studied.

One can contemplate that there are applications, however, where information on specimen geometry would be useful. This information can be expressed in terms of the characteristic delay times. It was shown that the cepstrum is most useful in detecting and measuring these characteristic times even in rather complex spectra. If analysis of the fine structure is considered, however, the first step is to eliminate or at least account for any fine structure produced from such factors as dry couplant pads. 


\section{APPENDIX A - THE SOURCE OF INTERFERENCE PEAKS IN THE MAGNITUDE OF THE FREQUENCY SPECTRUM} p. 8):

The magnitude of the frequency spectrum, $A(f)$, is given by, (ref. 17 ,

$$
A(f)=\frac{1}{2 \pi} \int_{-\infty}^{+\infty} x(t) e^{-i 2 \pi f t} d t
$$

Here $f$ is frequency, $t$ is time, and $x(t)$ is the time domain signal collected at the receiving transducer.

Let the time domain signal be a pulse arrival $x_{1}(t)$ and a pulse arrival $x_{2}(t)$ that are components of an AU signal.

$$
A(f)=\frac{1}{2 \pi} \int_{-\infty}^{+\infty}\left[x_{1}(t)+x_{2}(t)\right] e^{-i 2 \pi f t} d t
$$

Let the pulses be related by

$$
x_{2}(t)=C x_{1}(t-T)
$$

The two pulses have a common source, the input pulse from the sending transducer. $T$ is the characteristic time between them. Since they left the sending transducer at the same time, $T$ is the difference in their transit time from sender to receiver. The factor $c$ represents the difference in attenua$t$ ion between the pulses due to the longer time of travel for one of them. $C$ will therefore be frequency dependent. C must also contain any nontime dependent phase differences incurred by the pulses during the transit of the specimen. The only such phase differences, however, are at reflections. Reflections multiply the signal by plus or minus a positive constant. There fore the cumulative effect of all such reflections will be plus or minus a positive constant. Then $C$ can be expressed as:

$$
C= \pm K(f)
$$

Substituting equations (A4) and (A3) into equation (A2) the amplitude of the spectrum at any frequency of is:

$$
A(f)=\frac{1}{2 \pi} \int_{-\infty}^{+\infty}\left[x_{1}(t) e^{-i 2 \pi f t} \pm K(f) x_{1}(t) e^{-i 2 \pi f(t+T)}\right] d t
$$

or

$$
A(f)=\frac{1}{2 \pi} \int_{-\infty}^{+\infty} x_{1}(t)\left[1 \pm K(f) e^{-i 2 \pi f 1}\right] e^{-i 2 \pi f t} d t
$$

The term in the bracket of (A6) modulates the frequency spectrum of the $x_{1}(t)$ pulse. The condition for equation (1) of the ANALYSIS section, $T=n / f$, where $n$ as an integer reduces the bracket to:

$$
[1 \pm K(f)]
$$


In the case of equation (2) where $T=(n+1 / 2) / f$ the bracket becomes:

$$
[1 \mp K(f)]
$$

The sign in equation (A8) will always be opposite the sign in equation (A7). Peaks occur in the frequency spectrum where the plus sign occurs and troughs occur for the minus sign. 


\section{REFERENCES}

1. Vary, A.; and Bowles, K.J.: Ultrasonic Evaluation of the Strength of Unidirectional Graphite Polyimide Composites. Proceedings of the Eleventh Symposium on Nondestructive Evaluation, ASNT, San Antonio, TX, 1977, pp. 242-258. (NASA TM X-73646.)

2. Hayford, D.T.; Henneke, E.G., II; and Stinchcomb,W. W.: The Correlation of Ultrasonic Attenuation and Shear Strength in Graphite-Polyimide Composites. J. Compos. Mater., vol. 11, Oct. 1977, pp. 429-444.

3. Vary, A.; and Bowles, K.J.: Use of an Ultrasonic-Acoustic Technique for Nondestructive Evaluation of Fiber Composite Strength. Reinforced Plastics - Contact 78, Proceedings of the 33rd Annual Conference, Society of the Plastics Industry, New York, 1978, Section 24-A, pp. 1-5.

4. Vary, A.; and lark, R.F.: Correlation of Fiber Composite Tensile Strength with the Ultrasonic Stress Wave Factor. J. Test. Eval., vol. 7, no. 4, July 1979, pp. 185-191.

5 Williams, J.H., Jr.; and Lampert, N.R.: Ultrasonic Evaluation of Impact-Damaged Graphite Fiber Composites. Mater. Eval., vol. 38, no. 12, Dec. 1980, pp. 68-72.

6. Henneke, E.G., II, et al.: A Study of the Stress Wave Factor Technique for the Characterization of Composite Materials. NASA CR-3670, 1983.

7. Hemann, J.H.; and Baaklini, G.Y.: The Effect of Stress on Ultrasonic Pulses in Fiber Reinforced Composites. NASA CR-3724, 1983.

8. Wehrenberg, R.H., II: New NDE Technique Finds Subtle Defects. Mater. Eng., vol. 92, no. 3, Sept. 1980, pp. 59-63.

9. Serabian, S.: Composite Characterization Techniques: Ultrasonic. Mantech Journa1, vol. 10, no. 3, 1985, pp. 11-23.

10. Lorenzo, L.; and Hahn, H.T.: Damage Assessment by Acousto-U1trasonic Techniques in Composites. Report WU/CCR-86/2, Washington University, Apr. 1986. (Avail. NTIS, AD-Al68097.)

11. Govada, A.; Henneke, E.G.; and Talreja, R.: Acousto-U1trasonic Measurements to Monitor Damage During Fatigue of Composites. 1984 Advances in Aerospace Sciences and Engineering: Structures, Materials, Dynamics and Space Station Propulsion, U. Yuceoglu and R. Hesser, eds., ASME, 1984, pp. 55-60.

12. Henneke, E.G., II; and Duke, J.C., Jr.: Analytical Ultrasonics for Evaluation of Composite Materials Response, Part I: Physical Interpretation. Analytical Ultrasonics in Materials Research and Testing Conference, A. Vary, ed., NASA CP-2383, 1986, pp. 141-152.

13. dos Reis, H.L.M.; and Kautz, H.E.: Nondestructive Evaluation of Adhesive Bond Strength Using the Stress Wave Factor Technique. J. Acoustic Emission, vol. 5, no. 4, Oct.-Dec. 1986, pp. 144-147. 
14. Kautz, H.E.: Ultrasonic Evaluation of Mechanical Properties of Thick, Multilayered, Filament Wound Composites. NASA TM-87088, 1985.

15. Kautz, H.E.: Acousto-U1trasonic Verification of the Strength of Filament Wound Composite Material. NASA TM-88827, 1986.

16 Krautkramer, J.; and Krautkramer, H.: Ultrasonic Testing of Materials, Springer-Verlag, 1969, pp. 23-27.

17. Bendat, J.S.; and Piersol, A.G.: Engineering Applications of Correlation and Spectral Analysis. John Wiley and Sons, 1980, pp. 122-142, 181-185.

18. Childers, D. G.; and Durling, A. E.: Digital Filtering and Signal Processing, West Publishing Co., St. Paul, 1975, p. $433 \mathrm{ff}$.

19. Karagulle, H.; Williams, J.H., Jr.; and Lee, S.S.: Stress Waves in an Isotropic Elastic Plate Excited by a Circular Transducer. NASA CR-38\%, 1985.

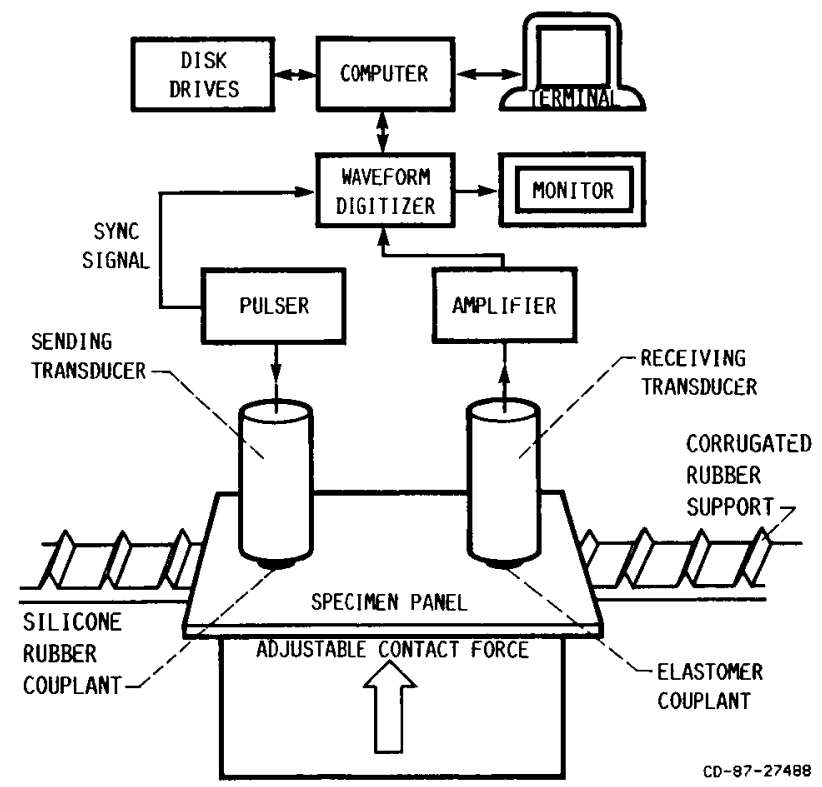

FIGURE 1. - BLOCK DIAGRAM OF THE ACOUSTO-ULTRASONIC AND DATA PROCESSING SYSTEM. 

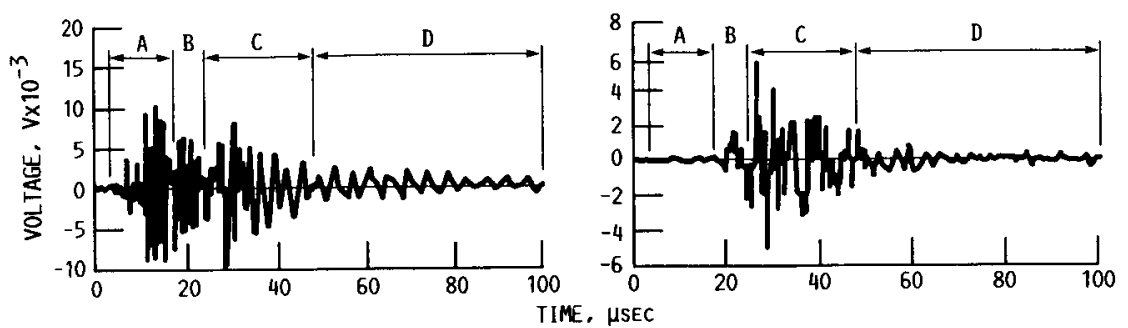

(A) COMPOSITE: TIME DOMAIN.

(B) RESIN ALONE: TIME DOMAIN.

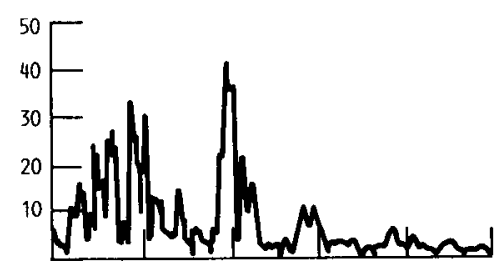

(C) COMPOSITE: TOTAL SPECTRUM.

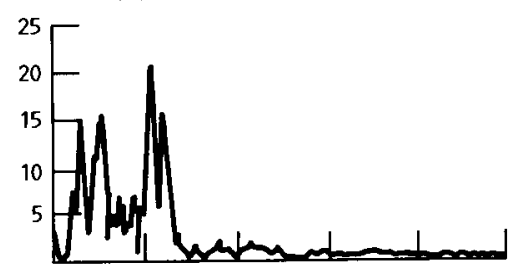

(D) RESIN ALONE: TOTAL SPECTRUM.
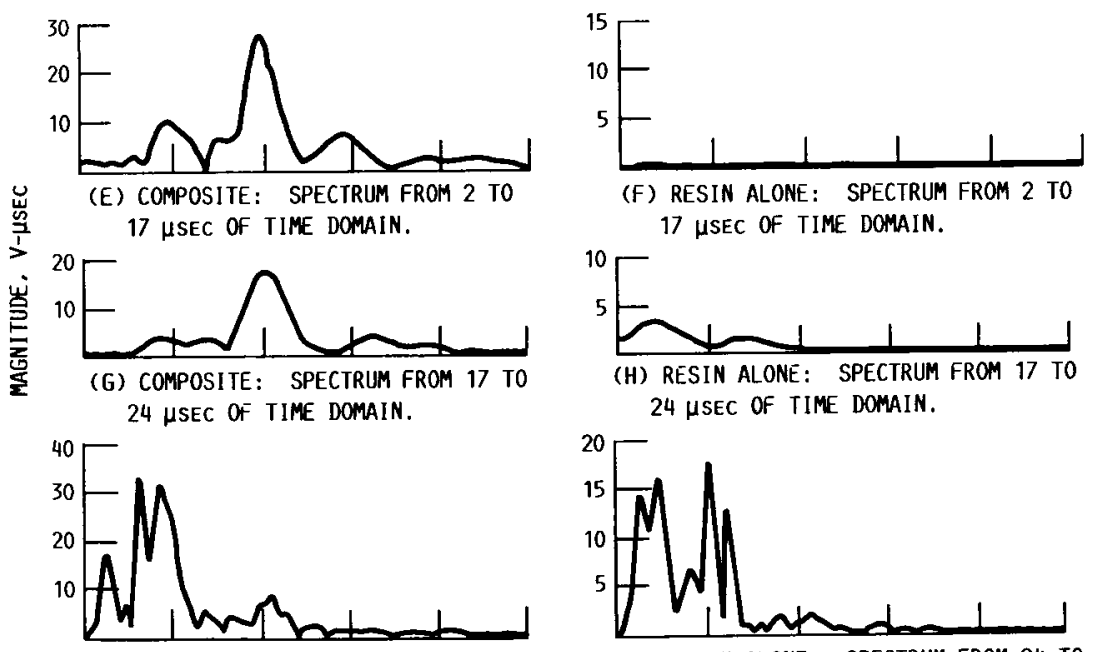

(I) COMPOSITE: SPECTRUM FROM 24 TO $48 \mu \mathrm{SEC}$ OF TIME DOMAIN.

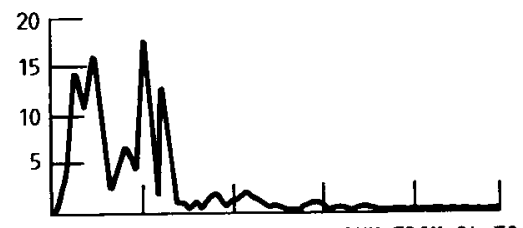

(J) RESIN ALONE: SPECTRUM FROM 24 TO 48 ISEC OF TIME DOMAIN.
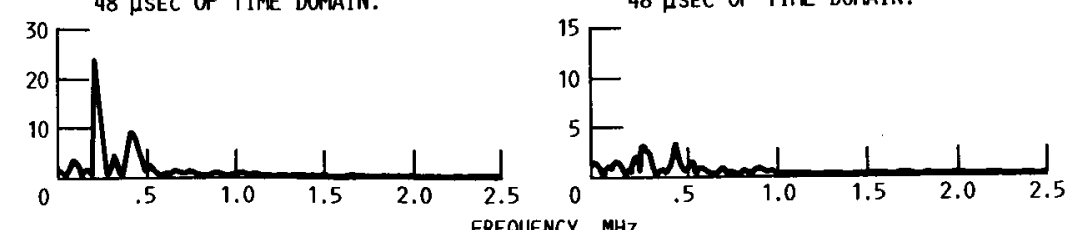

(K) COMPOSITE: SPECTRUM FROM 48 TO 100 HSEC OF TIME DOMAIN.

(L) RESIN ALONE: SPECTRUM FROM 48 TO 100 HSEC OF TIME DOMAIN.

FIGURE 2. - COMPARISON OF AU SIGNAL FROM GRAPHITE/EPOXY RESIN COMPOSITE PANEL WITH AU SIGNAL FROM PANEL OF THE RESIN ALONE. 


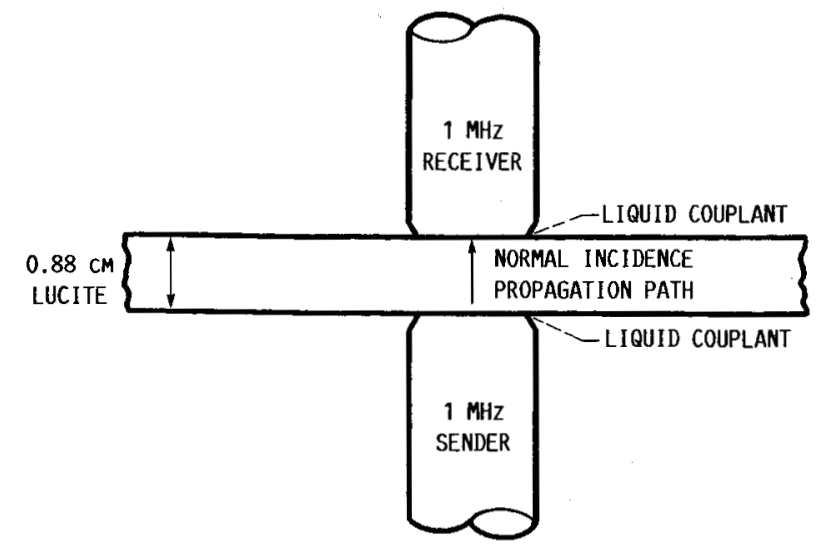

FIGURE 3. - TRANSDUCER ARRANGEMENT USED TO TRANSMIT ULTRASONIC WAVES DIRECTLY THROUGH A LUCITE PANEL AT NORMAL INCIDENCE.

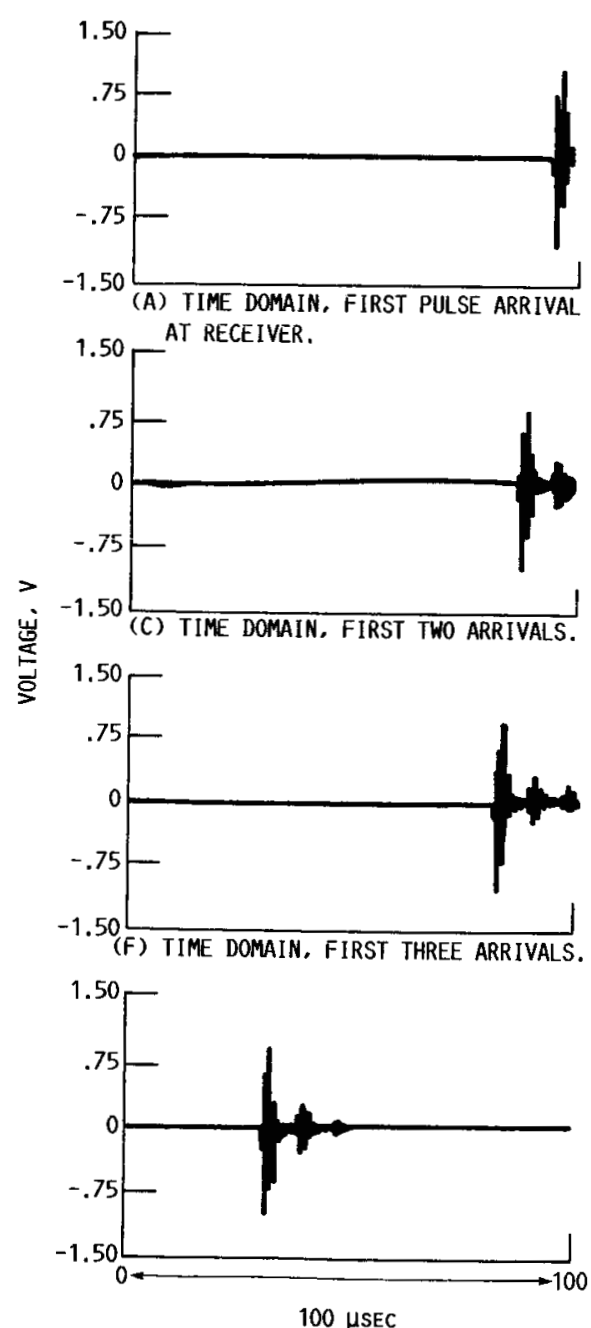

(I) TIME DOMAIN, ALL DETECTABLE
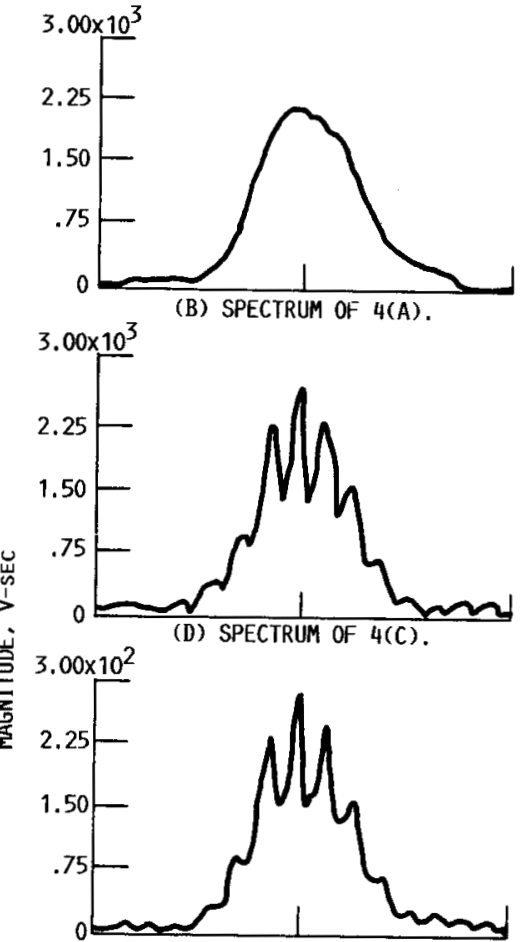

(6) SPECTRUM OF 4(F).

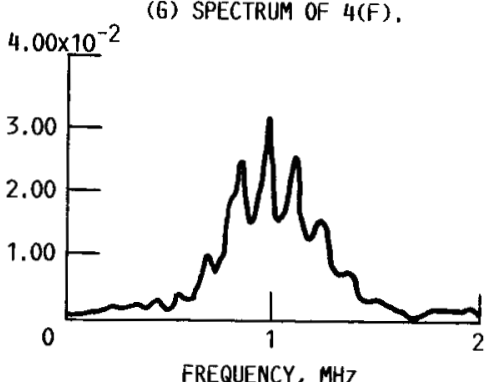

(d) SPECTRUM OF $4(\mathrm{I})$.
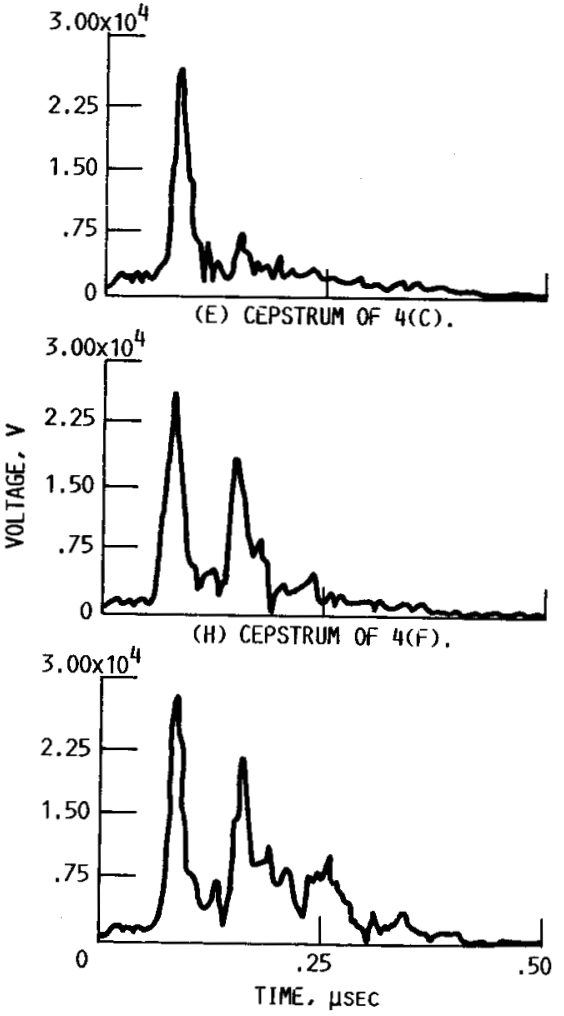

(K) CEPSTRUM OF $4(\mathrm{I})$. ARR IVALS.

FIGURE 4. - THROUGH-TRANSMISSION SIGNALS WITH 0.88-CM THICK LUCITE. LIQUID COUPLANT, AND TWO 1-MHZ TRANSDUCERS ARRANGED AS SHOWN IN FIGURE 3. 


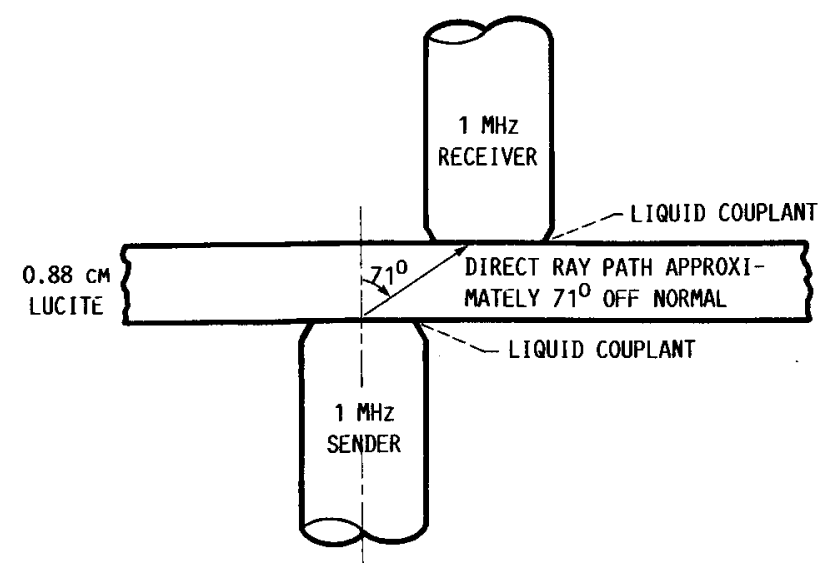

FIGURE 5. - OFFSET TRANSDUCER ARRANGEMENT USED TO TRANSMIT ULTRASONIC WAVES THROUGH LUCITE.

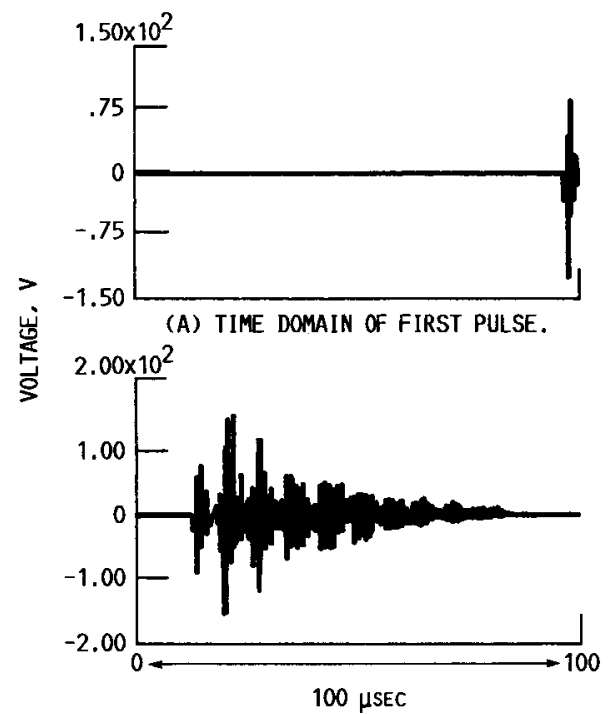

(C) TIME DOMAIN, TOTAL SIGNAL.

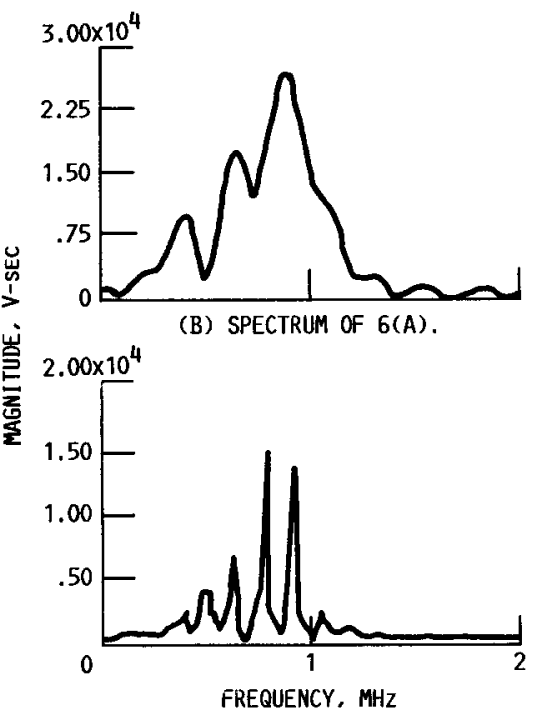

(D) SPECTRUM OF $6(C)$.

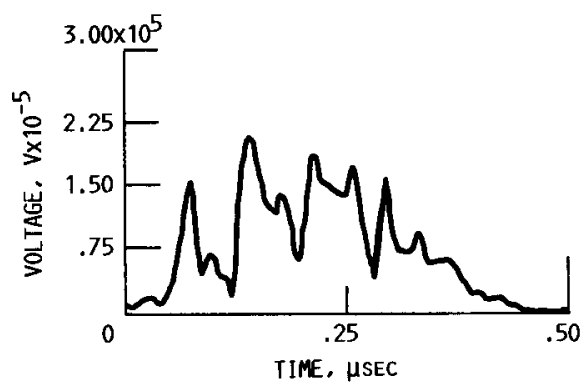

(E) CEPSTRUM OF $6(C)$. FIGURE 6. - THROUGH TRANSMISSION SIGNALS WITH THE TRANSDUCER ARRANGEMENT SHOWN IN FIGURE 5.

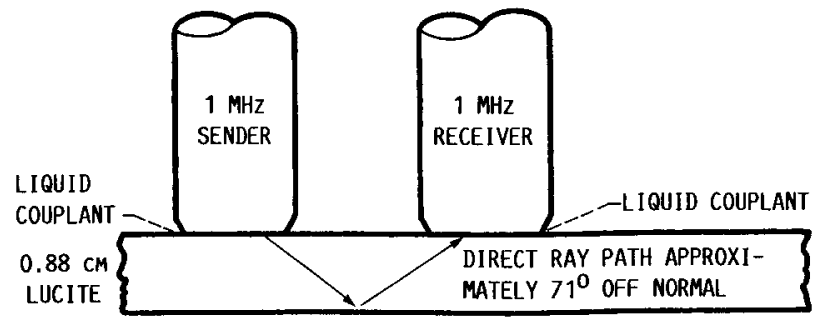

FIGURE 7. - TRANSDUCER ARRAMGEMENT USED TO TRANSMIT ULTRASONIC WAVES THROUGH LUCITE IN THE ACOUSTO-ULTRASONIC CONFIGURATION. 


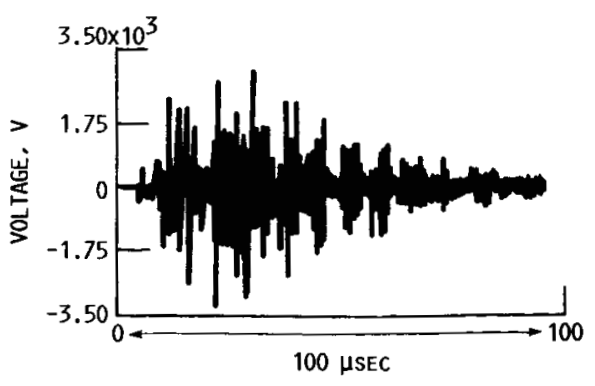

(A) TIME DOMAIN SIGNAL.

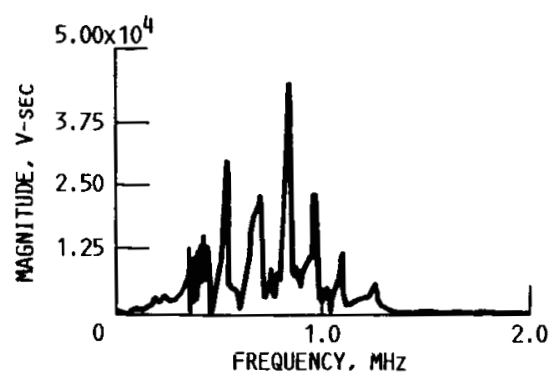

(B) SPECTRUM OF $9(A)$.

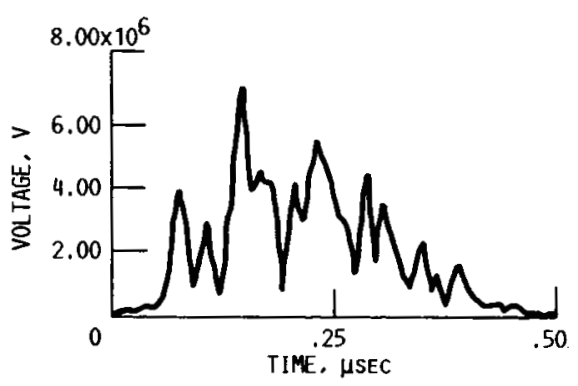

(C) CEPSTRUM OF $8(\mathrm{~A})$.

FIGURE 8. - ACOUSTO-ULTRASONIC SIGNAL KITH TRANSDUCER ARRANGEMENT OF FIGURE 7.

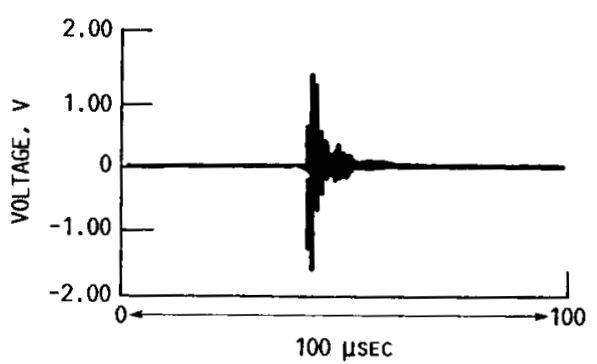

(A) TIME DOMAIN SIGNAL.

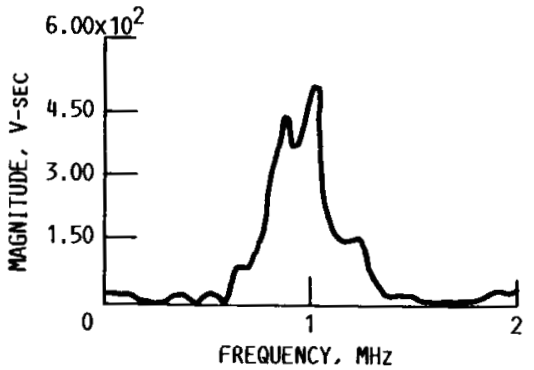

(B) SPECTRUM OF $g(A)$.

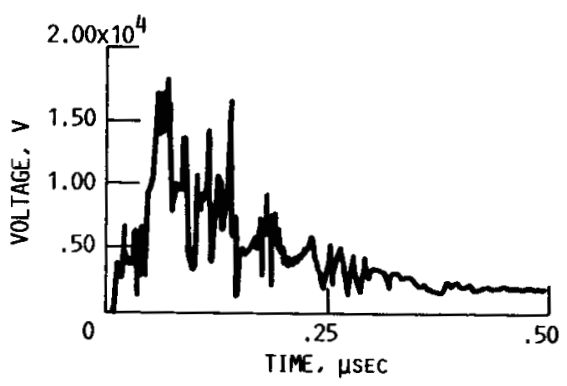

(C) CEPSTRUM OF $9(A)$.

FigURE 9. - THROUGH-TRANSMISSION WITH TWO 1-MHZ TRANSDUCERS AND ONE SILICONE RUBBER LAYER.

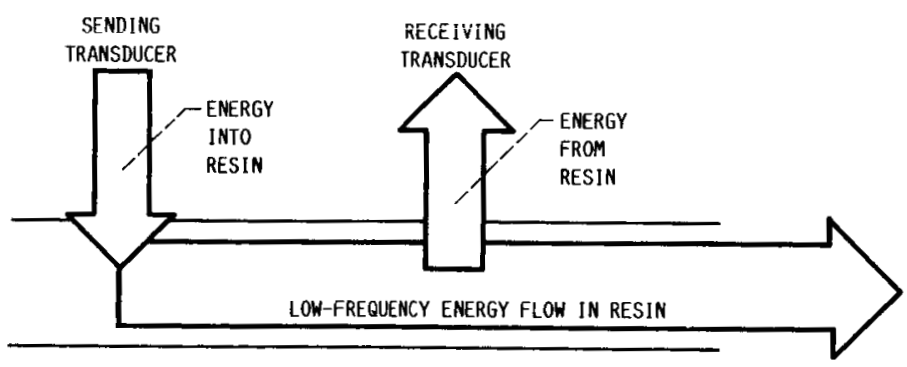

(A) FIBER-FREE RESIN PANEL.

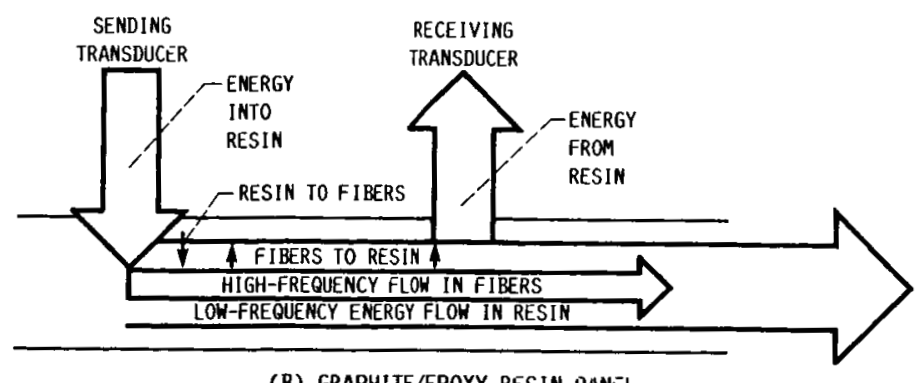

(B) GRAPHITE/EPOXY RESIN PANEL.

C0-87-27496

FIGURE 10. - ILLUSTRATION OF MODEL FOR ULTRASONIC WAVE MODES TO EXPLAIN ACOUSTO-ULTRASONIC RESULTS OBSERVED IN GRAPHITE/EPOXY LAMINATES. STRENGTH OF SIGNAL DIMINISHES (LEFT TO RIGHT) WITH DISTANCE FROM SOURCE. 


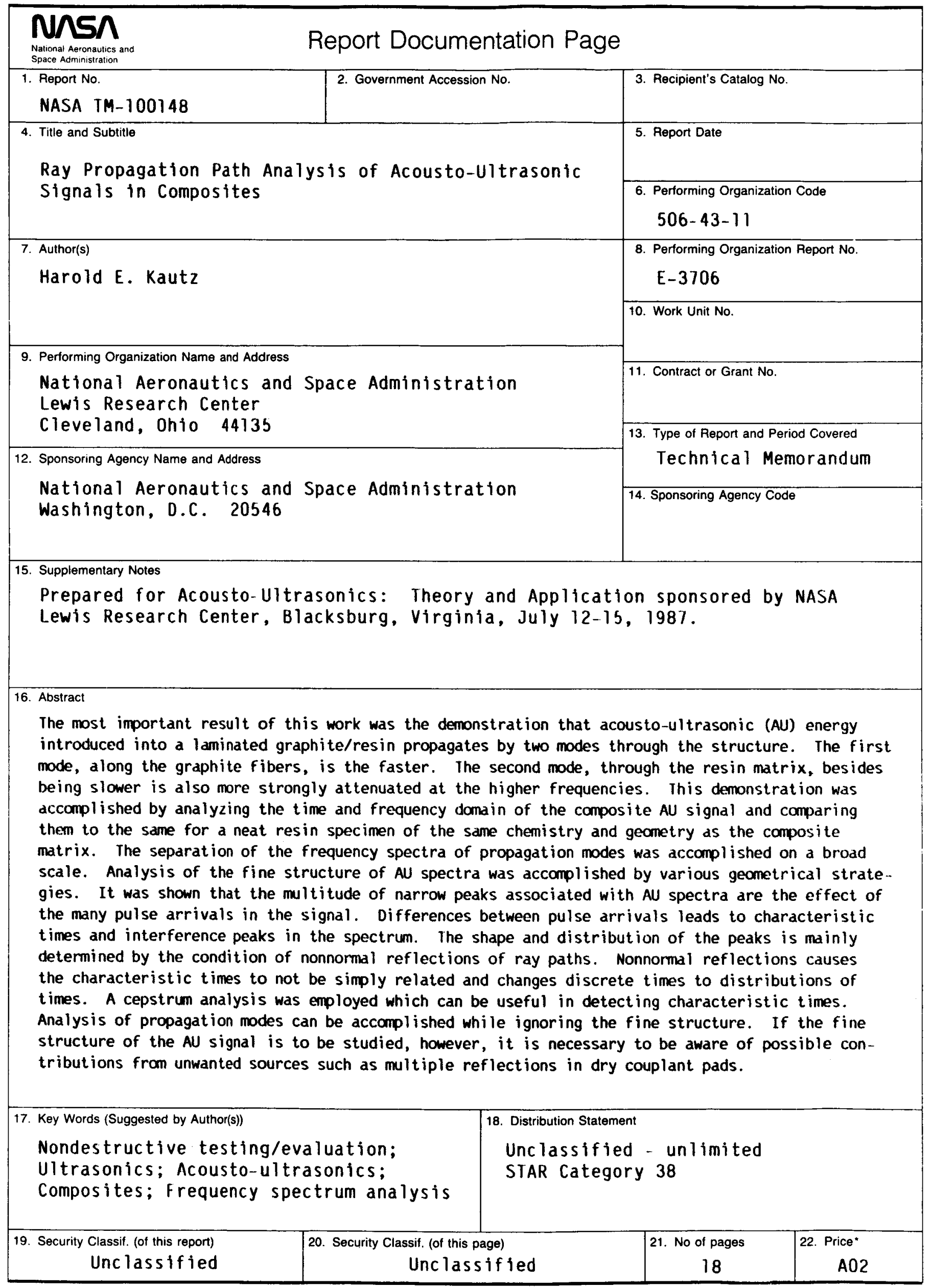

NASA FORM 1626 OCT $86 \quad$ "For sale by the National Technical Information Service, Springfield, Virginia 22161 